\title{
Fundamentos legales y teóricos en la creación de una cooperativa de ahorro y crédito en la comunidad de Mascarillas-Carchi (Ecuador)
}

\section{Yaćhaykunap kamachikuykunap takyachininkuna Illay mañakuy,ćhulay milachikunap allaykuyninka Maskarillas- Karchi malkaćhu (Ecuador)}

\author{
Osanenarotepage otikotagetiro patotagantsi paigiri \\ kirekipage kara timagantsi jitacha Maskarillas-Karchi \\ (Ecoador)
}

Recibido: 20 enero $2020 \quad$ Aprobado: 12 marzo 2020

Carlos Javier Lizcano Chapeta

Nacionalidad: Venezolana / Filiación: Universidad Regional Autónoma de los Andes (UNIANDES). Extensión Ibarra. (Ecuador). / Correo: lizcha_4@hotmail.com / ORCID: https://orcid.org/oooo-0002-1265-9465

Marco Patricio Villa Zura Nacionalidad: Ecuatoriana / Filiación: Universidad Regional Autónoma de los Andes (UNIANDES). Extensión Ibarra. (Ecuador). / Correo: ui.marcovilla@uniandes.edu.ec /ORCID: https://orcid.org/0000-0001-5248-0032

\section{Resumen}

El propósito de la investigación consistió en analizar los aspectos legales y teóricos que permiten la creación de una cooperativa de ahorro y crédito en la comunidad de Mascarillas, provincia del Carchi en Ecuador. Se utilizó una investigación de tipo descriptiva donde se plasmaron las características más importantes de los instrumentos jurídicos nacionales, y los aspectos teóricos para la creación de la cooperativa. Igualmente se empleó un diseño de investigación documental ya que se consultaron fuentes bibliográficas y digitales de segunda mano, así como leyes, teorías e instrumentos jurídicos relacionados con el tema. Las técnicas de análisis de datos que se utilizaron fueron el análisis de contenido y la hermenéutica que permitieron interpretar las informaciones recopiladas. Los resultados evidenciaron la necesidad de una entidad social que preste los servicios financieros con oportunidades para todos los habitantes del sector. Se concluyó que una nueva cooperativa promoverá una actividad de ahorro y crédito permitiendo generar el desarrollo económico de la población de Mascarilla y sus territorios aledaños.

\section{Palabras clave:}

Fundamentos, legales, teóricos, cooperativa, ahorro, crédito, Mascarillas.

\section{Lisichiku limaykuna:}

Takyaykuna, Kamachikukuna, yaćhaykuna, milachiku, milachi, mañana, Maskarillas

\footnotetext{
Nibarintsipage katingatsaro: kantëgotagetiro, otigobentagetiro, sangenarentsipage, patotagantsi paigiri kiregipage timaigatsi, Maskarillas.
} 


\section{Legal and Theoretical Foundations for the Creation of a Savings and Credit Cooperative in the Mascarillas-Carchi Community (Ecuador)}

\begin{abstract}
The purpose of the research was to analyze the legal and theoretical aspects that allow the creation of a savings and credit cooperative in the community of Mascarillas, province of Carchi in Ecuador. A descriptive investigation was used where the most important characteristics of the national legal instruments, and the theoretical aspects for the creation of the cooperative were reflected. Likewise, a documentary research design was used since second-hand bibliographic and digital sources were consulted, as well as laws, theories and legal instruments related to the subject. The data analysis techniques that were used were content analysis and hermeneutics that allowed interpreting the information collected. The results evidenced the need for a social entity that provides financial services with opportunities for all the inhabitants of the sector. It was concluded that a new cooperative will promote a savings and credit activity allowing the economic development of the population of Mascarilla and its surrounding territories to be generated.
\end{abstract}

\author{
Keywords \\ Fundamentals, \\ legal, theoretical, \\ cooperative, savings, \\ credit, Mascarillas.
}

\section{Fundamentos legais e teóricos na criação de uma cooperativa de poupança e crédito na comunidade Mascarillas-Carchi (Equador)}

\section{Resumo}

O objetivo da pesquisa foi analisar os aspectos legais e teóricos que permitem a criação de uma cooperativa de poupança e crédito na comunidade de Mascarillas, província de Carchi, no Equador. Utilizou-se uma investigação descritiva onde se refletiam as características mais importantes dos instrumentos jurídicos nacionais e os aspectos teóricos para a criação da cooperativa. Da mesma forma, foi utilizado um desenho de pesquisa documental porque foram consultadas fontes bibliográficas e digitais de segunda mão, bem como leis, teorias e instrumentos legais relacionados ao tema. As técnicas de análise de dados utilizadas foram análise de conteúdo e hermenêutica que permitiram a interpretação das informações coletadas. Os resultados evidenciaram a necessidade de uma entidade social que ofereça serviços financeiros com oportunidades para todos os habitantes do setor. Concluiu-se que uma nova cooperativa promoverá uma atividade de poupança e crédito que permitirá gerar o desenvolvimento econômico da população de Mascarilla e seus territórios circundantes.

\author{
Palavras-chave: \\ Fundamentos, \\ jurídicos, teóricos, \\ cooperativa, poupança, \\ crédito, Mascarillas.
}

\section{Datos de los autores}

Carlos Javier Lizcano Chapeta es Docente-Investigador de la carrera de Derecho en UNIANDES. Extensión Ibarra (Ecuador). Abogado por la Universidad de Los Andes (Venezuela). Licenciado en Educación por la Universidad Nacional Abierta (Venezuela) y Magister en Ciencias Políticas por la Universidad de Los Andes (Venezuela).

Marco Patricio Villa Zura es docente en la Universidad Regional Autónoma de los Andes, extensión Ibarra, Abogado de los Juzgados y Tribunales de la República por la Universidad Técnica Particular de Loja del Ecuador, Magister en Antropología por la Universidad FLACSO del Ecuador. 


\section{Introducción}

El Pueblo Afro ecuatoriano en el territorio ancestral Salinas-Concepción y Valle del Chota, desde los años sesenta comenzó a organizarse mediante la conformación de organizaciones sociales, lo cual nos ha permitido estar unidos y por ende buscar el fortalecimiento organizacional en el aspecto social, económico y político que nos sirve para satisfacer las necesidades de subsistencia cotidiana de cada familia.

En el actual momento el cooperativismo tiene una acción activa de consumo, ahorro y crédito que juega un papel trascendente en las comunidades rurales en el combate de la marginación y pobreza. Cabe mencionar que, en el territorio ya antes indicado, se impulsaron programas de reforma agraria sobre tenencia de la tierra normadas por el ex IERAC (hoy Subsecretaria de tierras) permitiendo la relevancia de cooperativas de producción y comercialización.

Las cooperativas de ahorro y crédito en el Ecuador constituyen un instrumento fundamental alternativo de desarrollo para los sectores rurales marginales, permitiendo generar créditos a las microempresas productivas, a los comerciantes y pequeños productores agrícolas como una respuesta de dignidad autogestionaria para dar solución a las necesidades colectivas.

Es de vital importancia indicar que, la Ley Orgánica de Economía Popular y Solidaria y del Sector Financiero Popular y Solidario se encuentra en vigencia a partir del año 2011.La forma de organización del Pueblo Afroecuatoriano es la que nos ha enseñado a gobernarnos asimismo en el ámbito político, público y privado, lo que nos permite estar altamente capacitados.

Es necesario en el momento actual, tener en consideración las políticas territoriales como el Plan de Desarrollo Estratégico de la parroquia de Mira, cantón Mira, provincia del Carchi, que se encuentran encaminados a la unión entre comunidades que permiten el fortalecimiento de la actividad de producción agrícola; se ha descubierto que la mayoría de los servicios de las instituciones financieras no pueden llegar a notable población compuesta por pequeños productores, comerciantes formales e informales, microempresarios y servicios, principalmente por los que habitan en la comunidad de Mascarilla, Parroquia Mira, cantón Mira, provincia del Carchi.

Este segmento de la población no tiene fácil acceso a los servicios que prestan las instituciones financieras ya que si lo hacen, estas operaciones se las realiza con un alto interés y este es un motivo para que los beneficiarios de dicho sector acudan a una zona de riesgo, a pesar del alto interés que cobran, dan una facilidad de crédito inmediato sin cumplir con muchos requisitos como lo solicitan las instituciones financieras.

Esta situación es la que limita solicitudes para el otorgamiento de crédito en general, pero particularmente, no permite el acceso al crédito a los habitantes que no poseen activos que sean aceptables como presentar garantías hipotecarias que pide el sistema financiero formal. Por esta situación es importante que se cree una cooperativa de ahorro y crédito para desarrollar al sector extemporáneo.

Observando las actividades de producción que se desarrolla en la comunidad de Mascarilla, parroquia de Mira, Cantón Mira, siendo una comunidad dedicada a la microempresa agrícola en diversidad de productos, pecuarios y servicios; donde se han evidenciado que la mayoría de los servicios ofertados por las instituciones financieras que se encuentran en la provincia del Carchi no pueden llegar a las personas de este sector poblacional. 
Considerando el constante crecimiento de la población y la dedicación permanente en sus actividades de producción por ende es necesario tomar en cuenta el sistema financiero cooperativista como una opción de desarrollo económico y social, y de este modo surge la necesidad de buscar una solución a la insatisfacción de las personas que pertenecen a las comunidades rurales marginales.

Dicho lo anterior, se pretende realizar un estudio que abarque las siguientes áreas: investigación sobre el servicio que ofrecería la futura cooperativa y el análisis de los servicios que presenta actualmente la competencia; un estudio técnico para la puesta en marcha; estudio de una base organizativa y legal acorde a la reglamentación de nuestro país, cuya finalidad será obtener resultados que ayuden a la estructuración de una cooperativa sólida con un pronto posicionamiento y con ciertas ventajas competitivas en el mercado de la ciudad.

Y es que en los últimos años se ha palpado la necesidad de cumplir con las expectativas del segmento de mercado seleccionado, en cuanto a obtener un servicio financiero el cual no ha sido satisfecho en gran parte por el sector financiero tradicional, tanto bancario como las cooperativas del Cantón de Mira, por esta situación, se crea la necesidad de constituir la cooperativa de ahorro y crédito en la comunidad de Mascarilla y de este modo desarrollar este sector de la sociedad que no ha sido atendido en estos aspectos.

\section{Metodología}

Durante el desarrollo de este estudio se utilizó una investigación de tipo descriptiva ya que se plasmaron las características y elementos más importantes de los aspectos teóricos para crear una cooperativa de ahorro y crédito en la comunidad de Mascarillas, Parroquia de Mira. Igualmente se establecieron algunos aspectos característicos de los nuevos enfoques legales sobre el cooperativismo.

Así mismo, se empleó un diseño de investigación documental ya que se consultaron fuentes bibliográficas y digitales de segunda mano, así como leyes, teorías e instrumentos jurídicos relacionados con el tema. En cuanto a las técnicas de recolección de la información, se aplicaron las fichas de contenido y los resúmenes, como estrategias para el acopio de la información más relevante, la cual suministró los datos fundamentales para cumplir con el propósito central de la investigación.

En lo que refiere a las técnicas de análisis de datos, se utilizaron estrategias como el análisis de contenido y la hermenéutica, las cuales permitieron interpretar las informaciones recopiladas. Finalmente, se utilizó el método crítico para plasmar las ideas y posturas propias sobre la normativa jurídica. Este proceso suministró insumos para plantear una solución al problema, mediante la creación de una cooperativa de ahorro y crédito, como alternativa estratégica de desarrollo social en los territorios de Mascarilla y sus alrededores.

\section{Resultados y discusión}

\section{Las cooperativas de ahorro y crédito}

Son organizaciones formadas por personas naturales y/o jurídicas que se unen voluntariamente con el objeto de realizar actividades de intermediación financiera y de responsabilidad 
social con sus socios y previa autorización de la Superintendencia, con clientes o terceros con sujeción a las regulaciones y a los principios reconocidos en la ley orgánica de la economía popular y solidaria y del sector financiero popular y solidario (Ley Orgánica de la Economía Popular y Solidaria del Sector Financiero Popular y Solidario).

En el mismo aspecto, la ley de Cooperativas, señala que: son cooperativas las sociedades de derecho privado, formadas por personas naturales o jurídicas que, sin perseguir finalidades de lucro, tienen por objeto planificar y realizar actividades o trabajos de beneficio social o colectivo, a través de una empresa manejada en común y formada con la aportación económica, intelectual y moral de sus miembros (Ley de Cooperativas del Ecuador).

En el actual momento es necesario que los grupos humanos busquen la integración con el fin de perdurar, competir, avanzar y desarrollarse, las cooperativas de ahorro y crédito, tienen el compromiso de crear nexos y acciones organizadas para mejorar en el aspecto social y económico de la familia de sus socios.

En las cooperativas de ahorro y crédito se ofrece la oportunidad de impulsar el desarrollo económico aplicando las metas establecidas en la institución, y proporcionar productos y servicios financieros de alta calidad a los clientes; la cooperativa antes mencionada proporciona a las socios acceso a servicios de ahorro muy necesarios, asegurando que sus ahorros estén depositados en entidades financieras que garantice seguridad.

En el mismo sentido Luis Chiriboga (2007) expresa que, la cooperativa es una sociedad de derecho privado, formada por personas naturales o jurídicas que sin perseguir finalidades de lucro, tienen por objeto planificar y realizar actividades o trabajos de beneficio social o colectivo a través de una empresa manejada en común y formado con la aportación económica, intelectual y moral de sus miembros.

\section{Investigación de mercado}

Según Nassir Sapag (2007) señala que, la investigación de mercado considera la opinión de los clientes como pertinente predictiva. Para ello, recurre a diversas formas de recopilación de sus opiniones, como por ejemplo: la toma de encuestas de una muestra representativa de la población, la realización de experimentos o la observación de los consumidores potenciales en mercados de prueba, entre otras, buscando probar o refutar hipótesis sobre un mercado especifico, es decir, las características de algún producto o de los consumidores.

Para Kinnear y Taylor (1998) indican que, "en el proyecto de la investigación de mercado se considera algunos pasos para ejecutar el proceso de investigación, es esencial prever todos los pasos y reconocer su dependencia".

\section{Análisis del entorno}

El entorno, es el estudio del lugar donde se va a constituir el proyecto, tomando en cuenta tanto los factores del macro-entorno como los del micro-entorno de la Comunidad de Mascarilla parroquia de Mira, cantón Mira, provincia de Carchi. 


\section{Cobertura de acción de la cooperativa de ahorro y crédito}

La cobertura de acción que realizará la cooperativa de ahorro y crédito Valle del Chota será el territorio ancestral Concepción-Salinas y Valle del Chota que pertenecen al Pueblo Afroecuatoriano.

\section{Mapa 1}

Asentamientos de las comunidades afro ecuatorianas en la Provincia del Carchi y Imbabura.

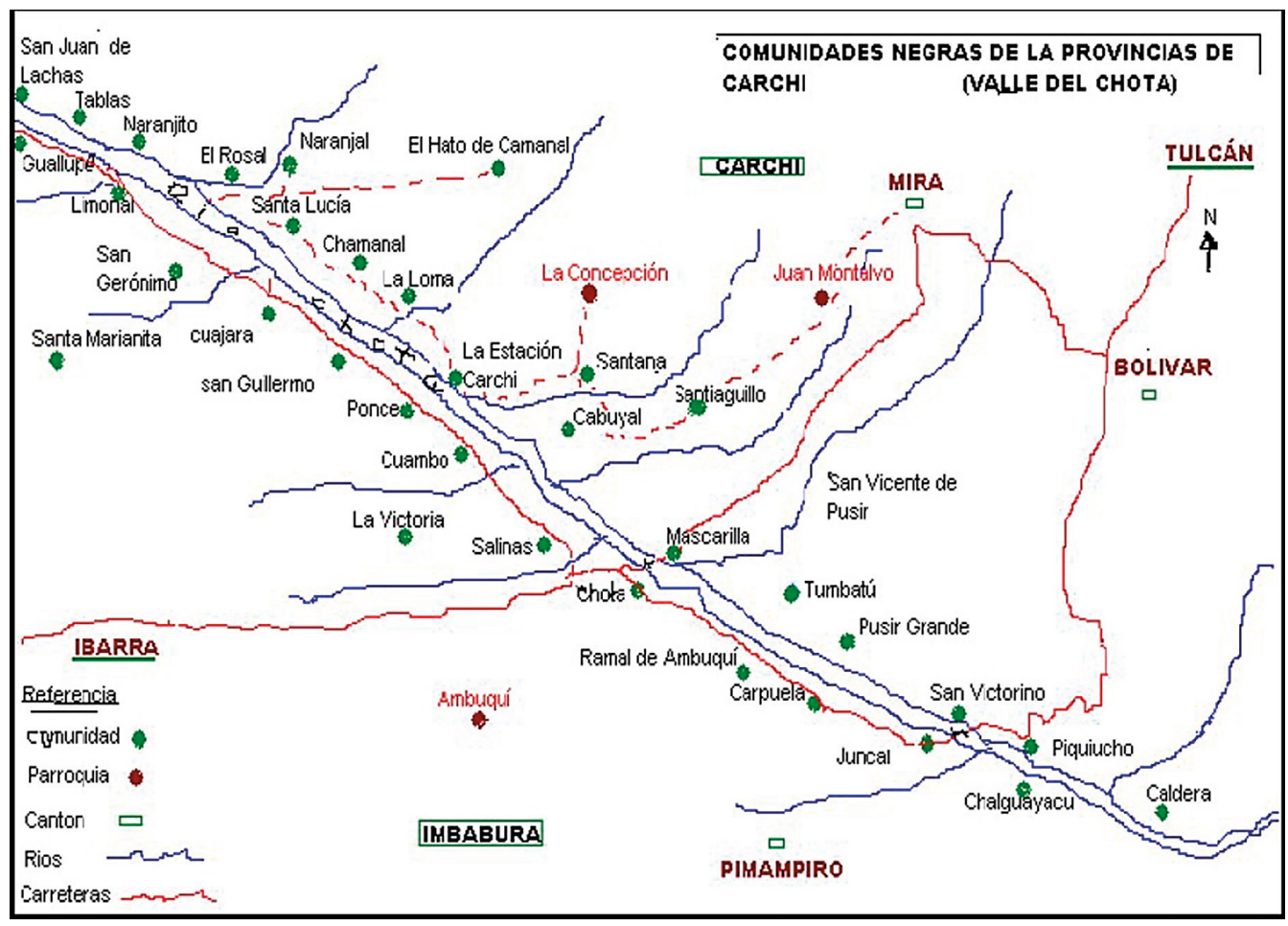

Fuente: Comunidades del Valle del Chota- Concepción y Salinas FECONIC (2014).

\section{Análisis del macro entorno}

La autora Pinargote Ángela (2014) expresa que, el macro-entorno está formado por fuerzas, las cuales son externas del mundo industrial que se pueden llegar a monitorear.

Comprende la influencia de los factores externos al sector industrial o al negocio a emprender, para realizar un análisis correcto se tomó en la cuenta dos puntos principales:

El análisis de mercado y, el del sector industrial, en donde se pretenda realizar el proyecto (Pinargote Ángela 2014: 8). 


\section{Análisis del micro-entorno}

En el mismo sentido la autora dice que, el micro-entorno, está conformado por el conjunto de fuerzas presentes en el horizonte inmediato, existen seis tipos; el propio negocio, proveedores, intermediarios, clientes, competidores y grupos de personas con intereses comunes. Esto ayuda a comprender la influencia de los factores que se encuentran al alcance de las organizaciones (Pinargote Ángela 2014: 9). Concluyo que, se ejecuta un análisis minucioso sobre el macro entorno basado en la influencia de los factores externos al negocio a emprender y el micro entorno el análisis del mercado y el del sector industrial.

\section{Participación Ciudadana}

Los ciudadanos de la comunidad de Mascarilla, que pertenecen a la Federación de Comunidades y Organizaciones Negras de Imbabura y Carchi “FECONIC" y de la Corporación Porvenir Valle del Chota participaran activamente en la creación de la nueva cooperativa de ahorro y crédito, permite que las personas se informen y opinen responsablemente acerca de la actividad financiera a desarrollarse.

La ciudadanía aporta con información relevante que sirve para recopilar ideas y realizar proyectos sostenibles que impulsan al desarrollo social, además son los que se encargan de revisar, evaluar y determinar si existe transparencia en la cooperativa de ahorro y crédito. Por ello, es importante recordar con Baca (2006), que "siempre que exista una necesidad humana de un bien o un servicio habrá necesidad de invertir, pues hacerlo es la única forma de producir un bien o un servicio" (p.2).

\section{Estudio Técnico}

Dentro de la viabilidad económica de un proyecto, este estudio tiene como objetivo dar respuesta al momento de cuantificar el monto de las inversiones y de los costos de operación pertinentes a esta área.

Es el análisis de elementos que tienen que ver con la ingeniería básica del producto y/o proceso que se desee implementar, para ello se tiene que hacer la descripción detallada del mismo con la finalidad de mostrar todos los requerimientos para hacerlo funcionar. Consiste en diseñar la función producción optima, que mejor utilice los recursos disponibles para obtener el producto deseado, sea este un bien o servicio (Sapag, 2007, p.32).

\section{Estudio Organizacional}

Dentro de los estudios de factibilidad de proyectos, este es el que tiene menos relevancia, y se refiere a los factores propios de la actividad ejecutiva de su administración: organización, procedimientos administrativos y aspectos legales (Sapag, 2008).

Dentro del estudio organizacional, está la de descripción de puestos, para esto se debe partir con la descripción del puesto. Y luego se comienza la búsqueda de la persona idónea para dicho puesto mediante entrevistas y revisando sus distintas aptitudes (Puchol, 2007). 
Dentro de una empresa se debe estudiar la cantidad de personas que se tendrán, cuantas son necesarias y cómo será su organización. Para esto se realizó un análisis de puesto de trabajo. Con la finalidad de obtener el personal más capacitado para la realización de las labores que se generaran dentro de la compañía, este consiste en la descomposición de las responsabilidades, tareas, características del entorno y las competencias del ocupante en unidades operacionales e identificables, se deberá escoger a la vez el proceso de análisis de puesto idóneo para el puesto de trabajo (Bustos, 2012).

Cuando el estudio organizacional no es lo suficientemente analítico se puede repercutir en la cuantificación incorrecta de aspectos tales como inversión inicial, capital de trabajo, y principalmente, en la elevación innecesaria de los costos administrativos. Para evitar este tipo de complicaciones, se recomienda la elaboración de este estudio a profesionales especializados en organización productiva y administrativa cuando se trate de una empresa grande y con una complicada estructura organizacional.

Cuando se realiza este tipo de estudios, es necesario explicar dicha estructura organizacional a través de organigramas, que facilitan la visualización y funciones del personal que haya definido para el funcionamiento de nuestro proyecto (Valbuena, 2000).

\section{Estudio de mercado}

En el presente estudio de investigación lo principal es la factibilidad del proyecto se especifica en el estudio de mercado, que significa conocer y cuantificar los clientes que pertenecen a la cooperativa en desarrollo, así como la competencia en este tipo de institución, un análisis de precios y la comercialización aplicar con el objeto de impulsar sus productos financieros.

Mediante el estudio de mercado se logra alcanzar lo siguiente:

a) Determinar los productos que se va a ofrecer

b) Analizar cuál es la demanda insatisfecha al cliente en el mercado

Por su parte Baca indica que, "El estudio de mercado se entiende como el área en que concluyen las fuerzas de la oferta y la demanda para realizar las transacciones de bienes y servicios a precio determinados" (2006, p.15).

Según Kotler expresa que, "la investigación de mercado consiste en el diseño, la recopilación, el análisis y el reporte de la información y de los datos relevantes del mercado" ( 2006, p.33).

En el presente proyecto de factibilidad el estudio de mercado se realizó en algunas instituciones privadas como cooperativas de ahorro y crédito, bancos comunitarios del Cantón Mira para determinar la oferta y demanda de los servicios y productos financieros que tiene la cobertura en el mercado.

Es importante indicar el estudio de la demanda de mercado se ejecutó al microempresario agrícola, al comerciante formal e informal de la comunidad de Mascarilla y el cantón Mira con el objeto de determinar cuál es la situación actual que tiene para acceder al financiamiento de créditos con las instituciones financieras locales para ejecutar la siembra de los productos.

Dentro de este estudio de investigación se logra determinar que el jefe de familia se dedica a la microempresa agrícola en el área superficial que oscila de 0.5 a 2.5 hectáreas de tierras que 
le sirve para ejecutar la actividad de producción agrícola, por una parte, la esposa del jefe de familia es una mujer idónea llena de cualidades y virtudes que se dedica a ser ama de casa y a la comercialización de los productos hacia el mercado local y nacional. Por otra, las mujeres de Mascarilla se dedican a la comercialización de productos del vecino país Colombia con la finalidad de ayudar y de esta manera salir adelante con su familia.

El micro empresario agrícola, el comerciante formal e informal en la comunidad de Mascarilla requiere financiamiento de un crédito a corto, mediano y largo plazo para realizar la inversión en la microempresa agrícola y en el negocio, una vez que la microempresa está en producción, el microempresario y el comerciante formal e informal realiza dos tipos de productos que son interesantes en el aspecto social; el primer producto es pagar el monto de crédito financiado por la cooperativa de ahorro y crédito en el tiempo acordado y el segundo producto es realizar depósito y ahorro a corto, mediano y largo plazo en la cooperativa antes indicada con el objeto de que el microempresario agrícola, el comerciante formal e informal sea estimulado de conformidad a la ley.

La investigación de mercado estuvo basada en la investigación de campo a través de entrevistas realizadas a personas que participan en las actividades de producción agrícola en la comunidad de Mascarilla, las entrevistas se ejecutaron a 100 personas donde se concluye lo siguiente:

a) De las personas encuestadas indican que, el sueldo oscila entre 100 y 400 dólares americanos.

b) Cabe mencionar que, en su mayoría las personas en la comunidad de Mascarilla ejecutan créditos pequeños desde 100 hasta 1000 dólares americanos.

c) De las personas entrevistadas en la comunidad de Mascarilla el $80 \%$ no tienen cuenta de ahorro y son quienes aceptan la creación de una nueva cooperativa de ahorro y crédito en la comunidad de Mascarilla.

d) De las personas encuestadas el $80 \%$ han considerado que los servicios financieros de las instituciones no otorgan créditos a las personas del sector Mascarilla.

e) El ahorro que realizarían los socios de la nueva cooperativa sería un promedio mensual de 10 a 30 dólares.

\section{¿Cómo se define al mercado?}

Según, Ficher y Espejo (2011) expresan que,

Es donde confluye la oferta y la demanda, en un sentido menos amplio, el mercado es el conjunto de todos los compradores reales y potenciales de un producto. Por ejemplo: El mercado de los autos está formado no solamente por aquellos que poseen un automóvil sino también por quienes estarían dispuestos a comprarlo y disponen de los medios para pagar su precio (p.87).

En el mismo sentido, Kotler, Philip (2007) señalan que: "Es un grupo de compradores y vendedores de un determinado bien o servicio. Los compradores determinan conjuntamente la demanda del producto, y los vendedores la oferta" (p.23).

\section{¿Cuál es la importancia del estudio de mercado?}

El estudio de mercado se constituye la base primordial para tomar la decisión de continuar, o no, con el estudio del proyecto de creación de la cooperativa de ahorro y crédito, ya que 
permite determinar si la creación de la nueva institución financiera cuenta con un mercado al cual es posible incursionar.

Por otra parte, el estudio de mercado provee de la información necesaria, con respecto a la demanda, la oferta; permitiendo de esta manera realizar un diagnóstico del mercado, para poder establecer las mejores condiciones de servicio financiero a través de la cooperativa de ahorro y crédito.

\section{¿Cuál es el objeto de estudio de mercado?}

El Estudio de Mercado representa una pieza fundamental que sirve en la elaboración de un proyecto de creación de la cooperativa de ahorro y crédito, porque es una herramienta por medio de la cual se obtiene la información necesaria y exacta que dará como resultado conocer los requerimientos y necesidades de los consumidores así como las fortalezas y debilidades de la competencia, con la finalidad y objeto primordial de saber la aceptación del producto en el mercado.

\section{Marco Legal}

La Constitución de la República, en su artículo 283, establece que el sistema económico es social y solidario; reconoce al ser humano como sujeto y fin; propende a una relación dinámica y equilibrada entre sociedad, Estado y mercado, en armonía con la naturaleza; y tiene por objeto garantizar la producción y reproducción de las condiciones materiales e inmateriales que posibiliten el buen vivir. Además, establece que el sistema económico se integrará por las formas de organización económica popular y solidaria, e incluirá a los sectores cooperativistas, asociativos y comunitarios.

Igualmente, en su artículo 309, establece que el Sistema Financiero Nacional incluye al sector financiero popular y solidario, el cual contará con normas y entidades de control específicas y diferenciadas, que se encargarán de preservar su seguridad, estabilidad, transparencia y solidez. Por su parte, en el artículo 311, establece que el sector financiero popular y solidario se compondrá de: cooperativas de ahorro y crédito, entidades asociativas o solidarias, cajas y bancos comunales, cajas de ahorro; estableciendo, que las iniciativas de servicios del sector financiero popular y solidario y las micro, pequeñas y medianas unidades productivas recibirán un tratamiento diferenciado y preferencial del Estado, en la medida en que impulsen el desarrollo de la economía popular y solidaria.

El Plan Nacional de Desarrollo 2007-2010 en su Objetivo No. 6 garantiza el trabajo, justo y digno, mediante la aplicación de la Política 6.1, cuyo objetivo es propiciar el empleo emergente y dinamizar la economía considerando, para tal efecto, las siguientes estrategias: 1. Incentivo a las Cooperativas de Ahorro y Crédito y entidades de Finanzas Solidarias para captar y canalizar el ahorro, con costos equitativos, favoreciendo en particular a los emprendimientos de la economía solidaria; 2 . Fomento a las micro y pequeñas empresas, tomando en cuenta a las Finanzas Solidarias en sus diversas formas, estimulando proyectos articuladores, cooperativos y comunales exitosos; y, 3. Consolidación de un programa integral dirigido a las Finanzas Solidarias en sus diversas formas, que permitan a micro y pequeños empresarios el acceso a recursos crediticios y mercados financieros bajo condiciones preferenciales. 
En este sentido, el Gobierno Nacional busca promover la democratización del acceso al crédito por parte de los actores de la Economía Popular y Solidaria, objetivo que será logrado con el impulso y fortalecimiento del sector financiero popular y solidario a fin de que sea un efectivo catalizador de recursos a los sectores más vulnerables del país. Igualmente, el sector cooperativo de ahorro y crédito ha posibilitado el acceso al crédito a los sectores de la economía popular que han sido excluidos del sector financiero formal, convirtiéndose así en un elemento dinamizador de la economía nacional, el aparato productivo en particular, fortaleciendo el tejido social en los territorios. Es necesario dotar a las cooperativas de ahorro y crédito de un marco jurídico que les permita desenvolverse dentro de los principios, valores y fines cooperativistas, en el marco de su objeto social de intermediario financiero cooperativo.

Por otra parte la Ley General de Instituciones del Sistema Financiero en su artículo 212 establece que mediante decreto ejecutivo se reglamentará la constitución, organización, funcionamiento y liquidación, así como las facultades de competencia y control de la Superintendencia de Bancos y Seguros respecto de las cooperativas de ahorro y crédito que realizan intermediación financiera con el público; y, En ejercicio de las atribuciones conferidas por los artículos 147 número 13 de la Constitución de la República del Ecuador, y 212 de la Ley General de Instituciones del Sistema Financiero.

\section{Conclusiones}

La carta magna del Estado indica que, el sector financiero popular y solidario se compondrá de cooperativas de ahorro y créditos, entidades asociativas o solidarias, cajas y bancos comunales, cajas de ahorro. Las iniciativas de servicios del sector financiero popular y solidario, y la micro, pequeñas y medianas unidades productivas, recibirán un tratamiento diferenciado y preferencial del Estado, en la medida en que impulsen el desarrollo de la economía popular y solidaria (Constitución de la República 2008, p. 311).

En la Constitución de la Republica establece que, se reconocen diversas formas de organización de la producción en la economía, entre otras las comunitarias, cooperativas, empresas públicas o privadas, asociativas, familiares, domésticas, autónomas y mixtas (Constitución de la República 2008: 319).

Por su parte, la ley de la Superintendencia de Economía Popular y Solidaria permite que se desarrolle económicamente la cooperativa de ahorro y crédito, se capacite a los socios de la cooperativa en normas y procedimientos Cooperativos.

Potenciar las prácticas de la economía popular y solidaria para que se desarrollen en las comunas, comunidades, pueblos y nacionalidades, y en sus unidades económicas productivas, va a permitir alcanzar el Sumak Kawsay (Ley Orgánica de Economía Popular y Solidaria del Sistema Financiero 2011, p. 3).

Finalmente, una nueva cooperativa promoverá una actividad de ahorro y crédito permitiendo generar desarrollo económico de la población de Mascarilla y el entorno. Estas instituciones tienen características de orden social y económico con el objeto de satisfacer las necesidades de las personas a un bajo costo. Es de vital importancia capacitar a los socios de la nueva cooperativa en el ámbito de cooperativismo y de este modo lograr el buen vivir. 


\section{Referencias bibliográficas}

Baca, G. (2006). Evaluación de proyectos.5a ed. México D.F., McGraw-Hill. 383p.

Constitución de la República del Ecuador (2008). Registro Oficial 449 del 20 de Octubre de 2008.

Bustos, F. (2012). Manual de instrumentos de gestión y desarrollo de las personas en las organizaciones. Madrid: Ediciones Díaz de Santos.

Chiriboga, L. (2007). Sistema Financiero. Primera Edición. Quito -Ecuador: Poligráficas Jokama.

Federación de Comunidades y Organizaciones Negras de Imbabura y Carchi (FECONIC). (2014).

Ficher, L. y Espejo, J. (2011). Mercadotecnia. México: Mcgraw-Hill/Interamericana Editores.

Kinnear, C.T. \& Taylor, R.J. (1998). Investigación de mercados. México: Mc. Graw Hill.

Kotler, P. (2007). Dirección de Mercadotecnia. Octava Edición. Editorial ESAN

Kotler, P Keller, K. (2006). Dirección de Marketing. 12a ed. México: Pearson Educación.

Ley de Economía Popular y Solidaria y del Sector Financiero Popular y Solidario (2011). Registro Oficial $\mathrm{N}^{\circ} 444$ - martes 10 de mayo del 2011.

Ley de Cooperativas del Ecuador (2001). Registro Oficial N400 del 29 de agosto de 2001.

Pinargote, A. (2014). Estudio de factibilidad para la creación de una cooperativa de ahorro y crédito en la Parroquia Crucita, Cantón Portoviejo, Provincia de Manabí. Tesis de grado para optar al título de maestría. Universidad de Guayaquil.

Puchol, L. (2007). Dirección y gestión de recursos humanos. Séptima edición. Madrid: Díaz de Santos.

Sapag, N y Sapag, R. (2008). Preparación y evaluación de proyectos. Quinta edición. Bogotá: McGraw-Hill.

Sapag, N. (2007). Proyectos de Inversión. Formulación y Evaluación. México: Pearson Prentice Hall. Secretaria Nacional de Planificación y Desarrollo (2007). Plan Nacional de Desarrollo 2007-2010. SEPLANDES-Ecuador.

Valbuena, R. (2000). La evaluación del proyecto en la decisión del empresario. México, CV Ediciones.

(C) Los autores. Este artículo es publicado por la Horizonte de la Ciencia de la Unidad de Posgrado de la Facultad de Educación de la Universidad Nacional del Centro del Perú. Este es un artículo de acceso abierto, distribuido bajo los términos de la Licencia Atribución-No Comercial 4.0 Internacional.(https://creativecommons.org/licenses/by-nc/4.o/), que permite el uso no comercial y distribución en cualquier medio, siempre que la obra original sea debidamente citada. 